\title{
LE APPLICAZIONI DELLE NUOVE MODALITÀ OPERATIVE MISTE AL RILIEVO DELLA GUGLIA MAGGIORE DEL DUOMO DI MILANO
}

\author{
CARLO MONTI (*) \\ Nota presentata dal m.e. Leone Corradi Dell'Acqua \\ (Adunanza del 26 giugno 2014)
}

SuNTO. - Le applicazioni delle nuove modalità operative miste al rilievo della Guglia Maggiore del Duomo da quota $63 \mathrm{~m}$ alla sommità della Madonnina e nei due altari laterali della campata di sinistra e di destra.

$* * *$

ABSTRACT. - The main purpose of the paper is to discuss how to use 3D models in the world of Cultural Heritage $(\mathrm{CH})$ by focusing on complex architectures. The experience of survey and modelling of the main spire of the Milan's Cathedral is described. It is as an example, how a detailed real based 3D model is created and used as base for an information system (BIM) that is now at disposal for the maintenance works of the cathedral. The interesting case of study is the chance to present common problems, adoptable solutions and to discuss about the use and implementation of this kind of system for $\mathrm{CH}$.

Forti cambiamenti ha subito anche quella che un tempo veniva chiamata fotogrammetria terrestre, ora fotogrammetria dei vicini. Uno degli esempi più interessanti nel nostro Paese è quello riguardante le applicazioni al rilevamento, a scopo di manutenzione e di catalogazione, di molti beni culturali. Un esempio concreto e importante è quello

(*) Politecnico di Milano, Dipartimento di Architettura, Ingegneria delle Costruzioni e Ambiente Costruito - A.B.C., 3D Survey Group, Milano, Italy.

E-mail: carlo.monti@polimi.it 
delle guglie, statue, archi rampanti, decori, altari del Duomo di Milano, dove si sono usati scansori laser e riprese fotografiche operando la metodologia del matching d'immagini (Figg. 1-12).

I sensori di rilievo di ultima generazione stimolano la ricerca scientifica degli addetti al settore e -viste le nuove possibilità- aumentano il livello di confronto pluridisciplinare e spingono verso la ricerca di nuovi utilizzi dei prodotti generati. Oggi le tecniche di rilievo permettono di costruire modelli 3D raffinati e i sistemi di Rapid Prototyping sono in grado di realizzare modelli fisici, con tempi e costi sempre più ridotti.

Nel settore dei beni culturali questa tecnologia rappresenta un mezzo efficace per la realizzazione di copie di reperti e parti di architetture; modello e copia fisica possono essere utilizzati per vari scopi:

- il modello costituisce la più moderna forma di catalogazione- archiviazione del bene (una banca dati 3D della forma);

- a partire dal modello 3D si possono generare tutte le viste e le sezioni desiderate, con un buon 3D si genera tutto il 2D necessario;

- sul modello si possono effettuare confronti (disponendo di diversi file) dello stesso oggetto ottenuti da rilievi effettuati in tempi diversi, per verificare lo stato di conservazione e per monitorare l'eventuale danneggiamento dovuto ad agenti esterni;

- dal modello si possono ricavare volume e dimensioni, ed è possibile inserire qualsivoglia tipo di informazione, anche collegamenti a DB d'archivio;

- il modello digitale può essere reso disponibile on-line e quindi navigato ed esplorato (gallerie virtuali, alto livello di fruibilità);

- il modello digitale permette operazioni di restauro virtuale (ricomposizione di frammenti, completamento di parti mancanti, ...);

- dal modello digitale (mediante tecnologie di rapid prototyping) si realizzano copie del bene;

- disporre di modelli digitali estende il settore delle attività dimostrative o didattiche, si possono, ad esempio, esplorare siti generalmente inaccessibili;

- modelli digitali e realtà virtuale offrono sensazioni molto coinvolgenti per gli spettatori.

Sicuramente l'estrema flessibilità delle tecnologie oggi disponibili applicate ai beni culturali offre modi nuovi per documentare, indagare, conoscere, diagnosticare, divulgare, fruire,.... migliorando il livello di conoscenza sugli oggetti e anche l'interesse da parte del pubblico che si avvicina con curiosità a queste nuove modalità di visualizzazione delle opere d'arte. 
In questo senso il lavoro in corso sul Duomo di Milano rappresenta un caso interessantissimo per le difficoltà che presenta e per le ricadute sia nel settore cantieristico di gestione del monumento che nelle aperte possibili applicazioni a livello museale.

Le immagini che accompagnano questa premessa danno ragione a quanto detto.

Vale la pena di parlare un poco più diffusamente di questo argomento, anche perché le attuali prestazioni degli scansori laser, integrate radiometricamente da prese fotografiche, trovano vaste applicazioni nell'ambito del rilevamento dei beni culturali in genere, delle architetture in particolare.

I "prodotti" del rilievo più richiesti nel campo dei Beni Culturali e Archeologici estesi sono oggi i seguenti:

- ortofoto: per la conoscenza del bene in primis e a cascata per il computo metrico estimativo;

- piante, sezioni, prospetti come base per le operazioni di restauro e manutenzione;

- modello tridimensionale globale del bene a una determinata data temporale.

L'integrazione di rilievo laser e fotogrammetria può fornire la soluzione ottimale per ottenere i suddetti prodotti in maniera rigorosa e con tempi rapidi.

Il risultato di una scansione laser è come si è detto a suo tempo, una nuvola di punti con coordinate XYZ molto densa, che costituisce da sola un modello tridimensionale completo e discreto, ma per la numerosità dei punti considerabile continuo (quindi finito senza alcuna elaborazione successiva) ad una certa scala.

Ai fini della completezza e della comprensione del modello tridimensionale l'integrazione dell'informazione di colore sulla nuvola di punti incrementa la leggibilità del dato e favorisce la sua interpretazione da parte dell'operatore (modello tridimensionale). Tre sono le tecniche ormai consolidate:

a) "intensity based";

b) "feature based";

c) "relational".

In a) le immagini originali vengono usate come matrici di grigio; il metodo è detto anche "area-based matching". L'incertezza è oggi subpixel e spesso minore sino a $1 / 10$ di pixel.

In b) il processo richiede per prima cosa le specificità delle imma- 
gini di base come contorni, angoli, areole, strutture e così via. L'incertezza è sub-pixel, ma minore del caso a). I procedimenti sono:

1) relazionali,

2) stime robuste,

3) cross-correlation,

4) programmazione dinamica,

5) correlazione fra elementi grafici.

In c) si usano relazioni geometriche o d'altro tipo fra strutture e caratteristiche del rappresentato. Il metodo non è molto accurato ma è robusto e non richiede elevate approssimazioni. Raramente viene però usato per generare DSM (Digital Surface Model).

La correlazione area-based è un metodo di applicazione locale basato sull'accoppiamento di due areole di pixels (una per ogni immagine). Per prima cosa, viene scelto un pixel $p$ di coordinate $(i, j)$ nella prima immagine. Questo pixel sarà il baricentro di una finestra di ricerca con dimensioni $\mathrm{N} \times \mathrm{N}$ (finestra di riferimento). Trovare il pixel della seconda immagine corrispondente a $p$ significa confrontare la finestra di riferimento con alcune finestre prese dalla seconda immagine (frames di ricerca). Una volta trovata la zona di ricerca che più assomiglia alla finestra di riferimento, il baricentro di tale finestra sarà il pixel corrispondente a $p$.

Visto in termini assolutamente generali, il problema ha una complessità molto elevata ed è intrattabile: esso va, dunque, semplificato. L'introduzione del vincolo epipolare è un'ottima soluzione al fine di semplificare il problema iniziale. Questo vincolo impone che, nelle immagini stereoscopiche, punti corrispondenti debbano sempre giacere su linee epipolari. Queste linee corrispondono all'intersezione di un piano epipolare (il piano contenente un punto dell'oggetto nell'immagine 3D e i punti principali dei due obiettivi) con i piani di immagine sinistro e destro. La ricerca delle corrispondenze, quindi deve essere effettuata solo su tali linee epipolari e non sull'intera immagine. L'utilizzo del vincolo epipolare riduce il problema, inizialmente bidimensionale, a uno monodimensionale, poiché il pixel obiettivo nella seconda immagine deve trovarsi sulla linea orizzontale di ordinata uguale a quella del pixel di partenza (per l'appunto, linea epipolare).

Un modo semplicistico di trovare la disparità è quello di cercare lungo le linee epipolari il massimo della funzione di correlazione; tuttavia si può fare molto meglio adoperando dei vincoli aggiuntivi: 
1) unicità: un punto in una immagine può corrispondere al massimo ad un punto nell'altra immagine,

2) continuità a tratti delle superfici nella scena: il fatto che il mondo solitamente è continuo a tratti, implica che punti vicini di una scena abbiano valori di profondità, e quindi di disparità, simili.

Oltre a questi vincoli, si possono trovare funzioni euristiche atte a migliorare l'algoritmo, come ad esempio l'introduzione di valori di soglia o l'analisi dell'andamento dei vari picchi che contraddistinguono la funzione di correlazione.

In definitiva, gli algoritmi di autocorrelazione permettono il riconoscimento di segnali o forme atte a individuare automaticamente punti omologhi; ciò permette di limitare l'impiego dell'operatore umano e in certi casi di sostituirlo.

Torniamo alla procedura "area based"; di fatto si tratta di un confronto statistico fra i valori di grigio di due minuscole porzioni d'immagine (ormai solo digitali) di cui la prima è detta "matrice sagoma" (search window) e la seconda "matrice di ricerca" utilizzando algoritmi di "cross-correlation" e dei minimi quadrati ("least square modelling"). Viene qui ricercata la massima correlazione fra i livelli di grigio della matrice sagoma rispetto alla matrice di ricerca, assumendo come posizione corretta quella corrispondente al valore massimo del coefficiente di correlazione, che è dato da:

$r=\frac{\sigma_{12}}{\sigma_{1} \sigma_{2}}$

ove $\sigma_{1}$ e $\sigma_{2}$ sono gli sqm delle due matrici, mentre $\sigma_{12}$ è la covarianza. L'espressione di $r$ è la seguente:

$$
r=\frac{\sum_{i=1}^{n}\left(g_{1 i}-g_{1}\right)\left(g_{2 i}-g_{2}\right)}{\sqrt{\sum_{i=1}^{n}\left(g_{1 i}-g_{1}\right) 2 \sum_{i=1}^{n}\left(g_{2 i}-g_{2}\right) 2}}
$$

ove gli elementi sono i seguenti:

$g_{1 i}=$ livelli di grigio matrice sagoma in $i$

$g_{2, i}=$ livelli di grigio matrice di ricerca

$g_{1}, g_{2}=$ media livelli di grigio nelle due matrici

$n=$ numero totale pixel 
La trasformazione fra le due matrici è di tipo affine, a sei parametri e l'equazione relativa è la seguente:

$g_{1}(x, y)=g_{1} g_{2}(a x+b y+c d x+e y+f)+s$

Ecco la successione delle operazioni nel caso "area based":

- si seleziona l'entità da correlare nella prima immagine,

- si individua l'entità corrispondente sulla seconda immagine,

- si calcola la posizione del punto da correlare,

- si valuta la qualità della correlazione effettuata.

Per la prima operazione si impiegano algoritmi detti genericamente "operatori".

Gli operatori sono quindi algoritmi per l'estrazione delle diverse immagini di punti che sono potenzialmente degli adatti candidati alla correlazione. Adatti candidati di punti omologhi sono immagini di pixel di caratteristiche tali, da essere uniche entro limitati intorni, che sembrano avere similari aspetti nelle corrispondenti immagini. L'operatore determina per ogni pixel uno o più parametri per il calcolo di un dato valore da utilizzare nella successiva correlazione.

Esamineremo qui due operatori: quello di Moravec e quello di Förstner, a titolo di esempio. Il primo calcola le somme quadratiche dei gradienti in quattro direzioni principali della finestra d'immagine esaminata. Il secondo calcola la matrice di covarianza dello spostamento di finestra immagine. Ecco le espressioni relative ai due operatori.

\section{Operatore di Moravec}

$$
\begin{aligned}
& V_{1}=\frac{1}{p(q-1)} \sum_{j=-k}^{+k} \sum_{j=-1}^{+l-1}[g(i, f)-g(i, f+1)]^{2} \\
& V_{2}=\frac{1}{(p-1) q} \sum_{i=-k}^{+k-1} \sum_{j=-1}^{+l}[g(i, f)-g(i+1, f)]^{2} \\
& V_{3}=\frac{1}{(p-1)(q-1)} \sum_{i=-k}^{+k-l} \sum_{j=-1}^{+l-1}[g(i, j)-g(i+1, j+1)]^{2} \\
& V_{4}=\frac{1}{(p-1)(q-1)} \sum_{i=-k}^{+k-l} \sum_{j=-1}^{+l-1}[g(i, j+1)-g(i+1, f)]^{2}
\end{aligned}
$$

essendo: $V=\min \left(V_{1}, V_{2}, V_{3}, V_{4}\right)$ mentre: $p=2 k+1$ e $q=2 l+1$ 
Il programma dell'operatore di Moravec è di facile implementazione e relativamente veloce per i tempi d'impiego; non è però invariante alle rotazioni e la sua incertezza è di un pixel.

Vediamo ora l'operatore di Förstner.

Questo operatore è fondato sul principio che la zona intorno ad un punto $f(x, y)$ è una copia slittata e affetta da rumore del segnale originale dell'immagine $g(x, y)$ :

$f(x, y)=g\left(x+x_{0}, y+y_{0}\right)+e(x, y)$

linearizzando e ponendo $x_{0}=y_{0}=0$ si ha:

$d g(x, y)-e(x, y)=\partial g / \partial x x_{0}+\partial g / \partial y y_{0}=g_{x} x_{0}+g_{y} y_{0}$

essendo:

$d g(x, y)=f(x, y)-g(x, y)$

Se ne ottiene la matrice:

$\left|\begin{array}{cc}\sum g_{x}^{2} & \sum g_{x} g_{y} \\ \sum g_{y} g_{x} & \sum g_{y}^{2}\end{array}\right|\left|\begin{array}{l}x_{0} \\ y_{0}\end{array}\right|=\left|\begin{array}{ll}g_{x} & d g \\ g_{y} & d g\end{array}\right|$

Che si può scrivere come:

$N x=A^{T} A x=A^{T} d g$

Le relazioni sopra viste sono la base sulla quale sono maturati sofware numerosi di trattamento sia delle nuvole laser, sia del matching d'immagine. La potenza dei calcolatori attuali, portatili compresi, permettono di orientare centinaia di immagini trovando i punti corrispondenti e costruire quindi una nuvola così fitta che costituisce di fatto un modello digitale misurabile e conosciuto nelle sue caratteristiche di posizione, forma e colore. I modelli 3D sono uno degli strumenti di conoscenza oggi disponibili, la cui realizzazione è in continua crescita e sostenuta dall'aumento delle capacità hardware e software. La realizza- 
zione di modelli digitali, in particolare nel settore dei Beni Culturali, è comunque un processo abbastanza complesso che prevede l'impiego di strumenti diversi e programmi adeguati per le elaborazioni e necessita sempre dell'intervento di personale qualificato, sia durante le campagne di rilievo che nella fase di modellazione digitale. Sono disponibili sul mercato diversi tipi di sensori per il rilievo tridimensionale e gli strumenti maggiormente impiegati nel settore dei Beni Culturali sono gli strumenti laser scanner e le camere digitali.

Se oggi gli strumenti disponibili sono tanti e diversi bisogna però ricordare che la forma e la collocazione dell'oggetto da rilevare influenzano le operazioni di acquisizione.

Di fatto non esiste una metodologia di acquisizione che possa essere applicata in modo indifferenziato; la complessità della forma dell'oggetto, la sua dimensione e la sua posizione, le caratteristiche della superficie (texture) rendono ogni caso "un caso a sé" ed è l'abilità dell'operatore che rende possibile un risultato di qualità (oltre alle problematiche legate al tempo a disposizione per la campagna di rilievo e i costi sostenibili dal progetto).

\section{RINGRAZIAMENTI}

Un particolare ringraziamento per la collaborazione continuativa e la disponibilità assoluta al Direttore della VFD Dott. Ing. Benigno Mörlin Visconti Castiglione, al personale tutto e in particolare a Franco Aquilano, Massimiliano Regis. 


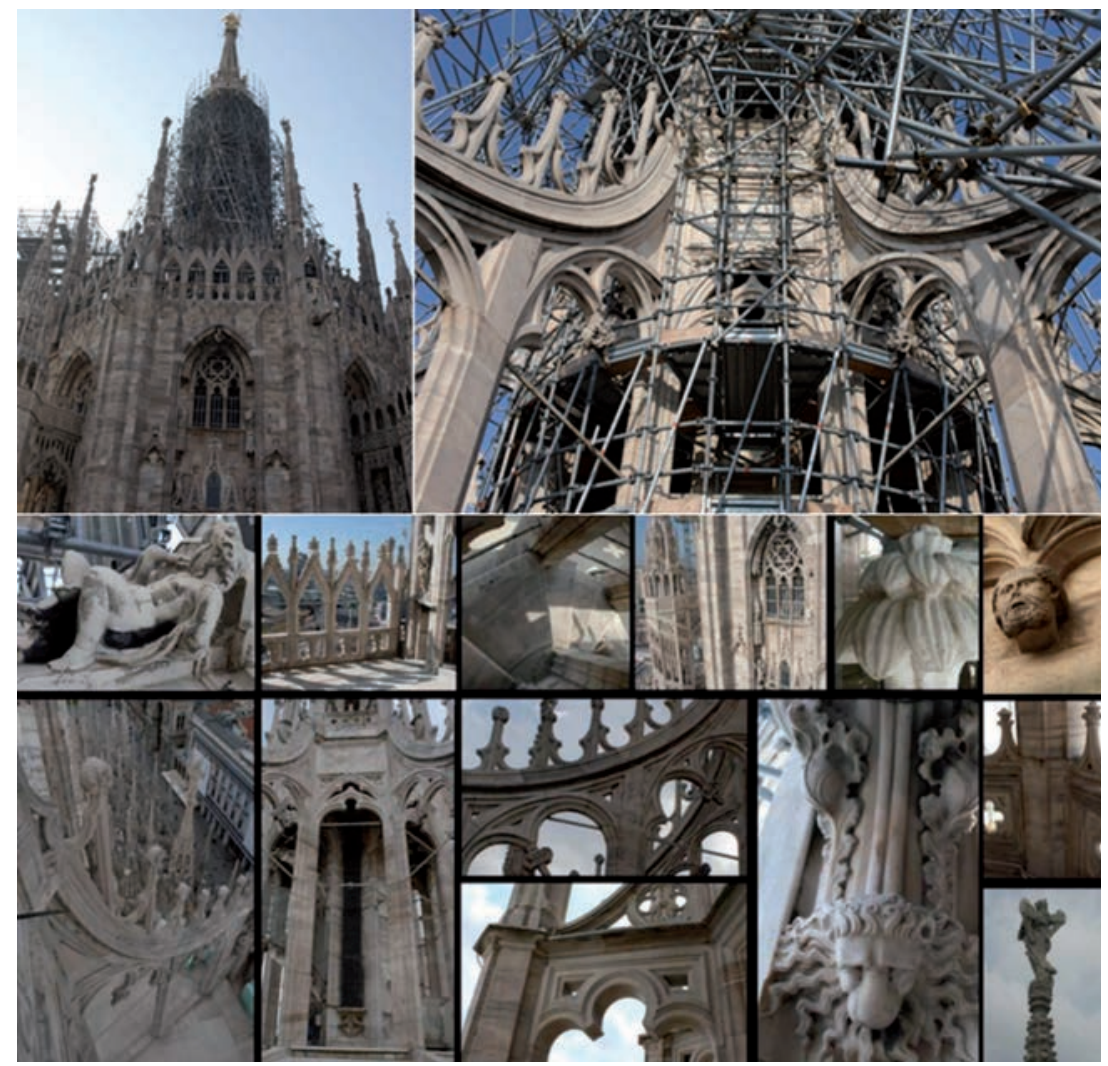

Fig. 1 - Il cantiere della Guglia Maggiore del Duomo di Milano. Il ponteggio strallato per le operazioni necessarie (controllo degli elementi marmorei, restauro, sostituzioni dei conci ammalorati, ....) è particolarmente complesso e ba richiesto un studio preliminare per verificare che lo stesso non gravasse sulla parte strutturale da quota 63 m fino alla sommità della Guglia a quota $108 \mathrm{~m}$. 


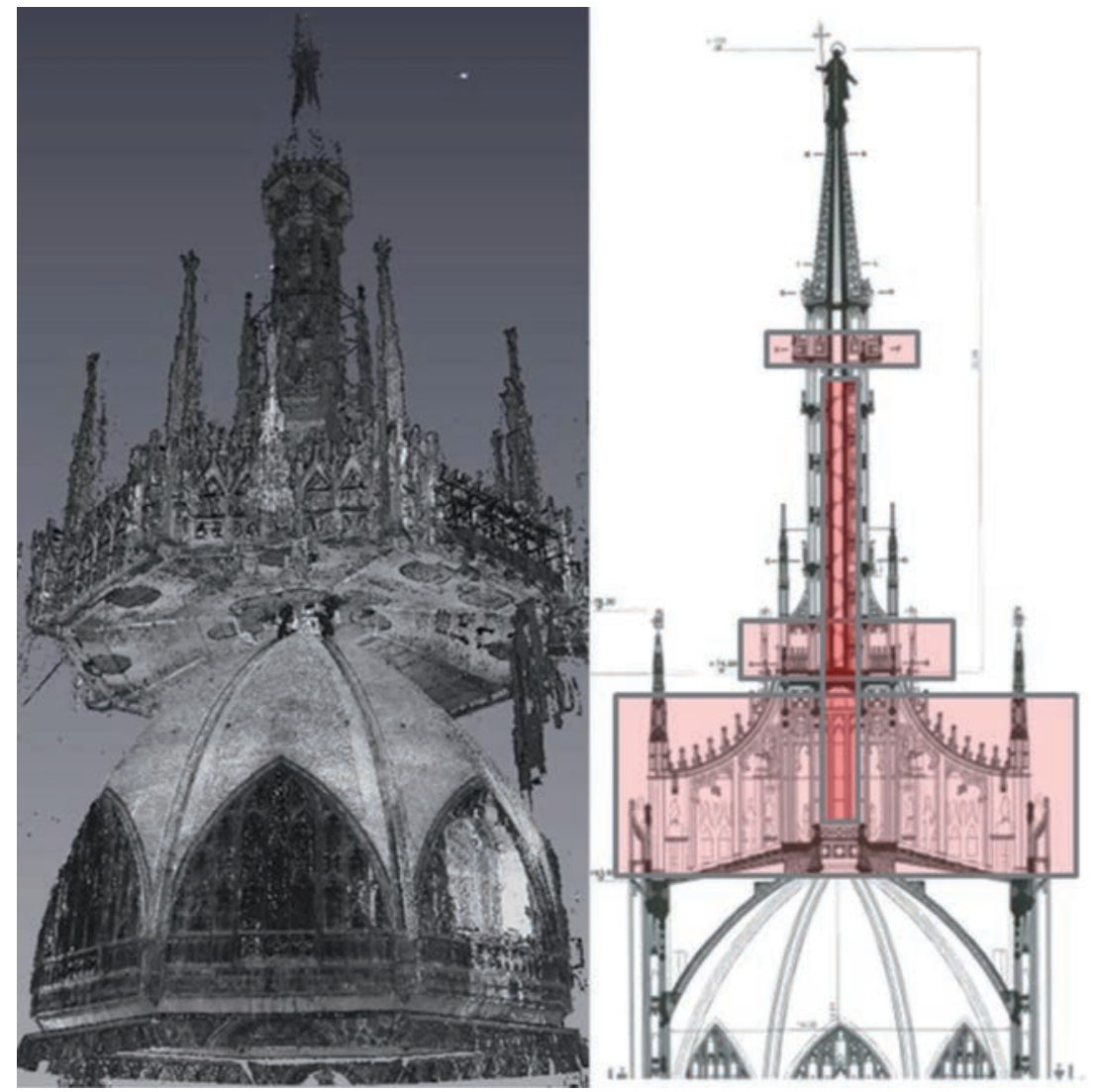

Fig. 2 - Qui si può vedere il rilievo 3D ottenuto col Laser Leica HDS 7000 con la visualizzazione delle nuvole di punti acquisiti georeferenziate.

Sono state effettuate 73 stazioni per un modello 3D composto da circa 600 milioni di punti. A destra si può vedere il passaggio dal 3D al 2D evidenziando partendo dall'alto il Belvedere, la scala alta e bassa, il belvedere basso e infine gli Archi rampanti e il Tiburio. 


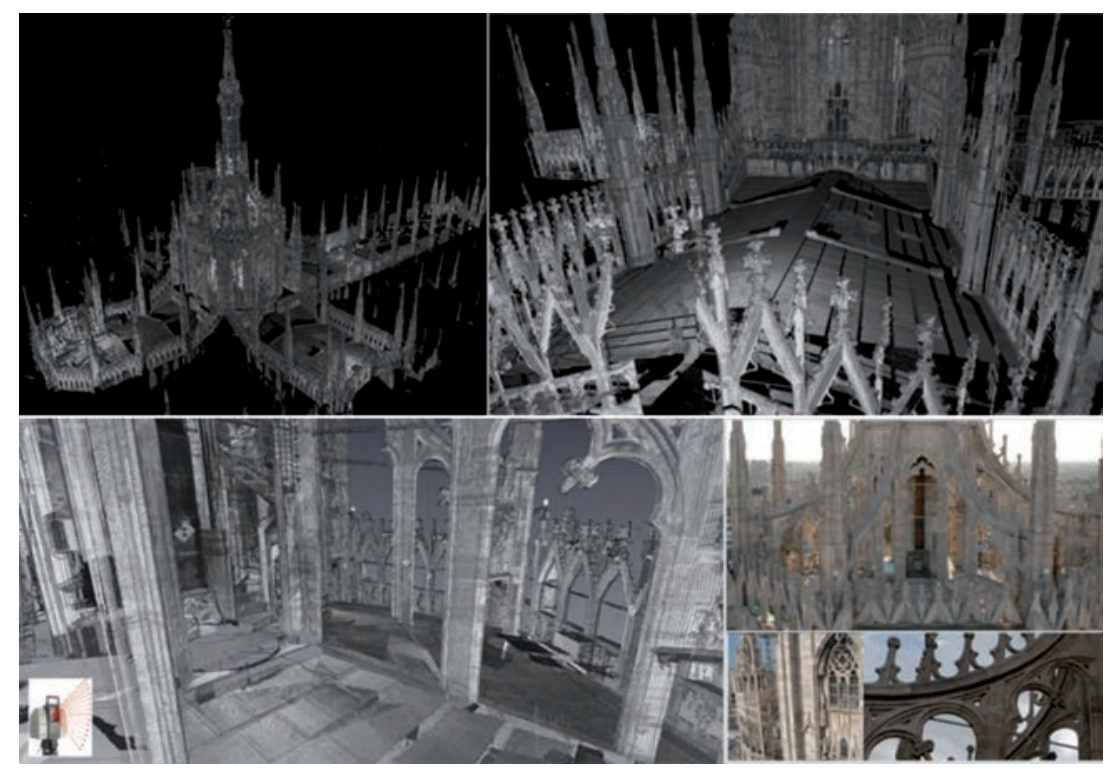

Fig. 3 - Come detto è stato realizzato un rilievo laser scanner dell'intero complesso del tiburio, la georeferenziazione delle nuvole ha permesso di ricavare un ricco modello - in formato nuvola di punti - utile per la costruzione del modello 3D e la georefenziazione delle singole parti sempre con laser Leica HDS 7000. Nella figura si vedono le estrazioni di zone di interesse creando delle "finestre" dal totale della nuvola di punti. 


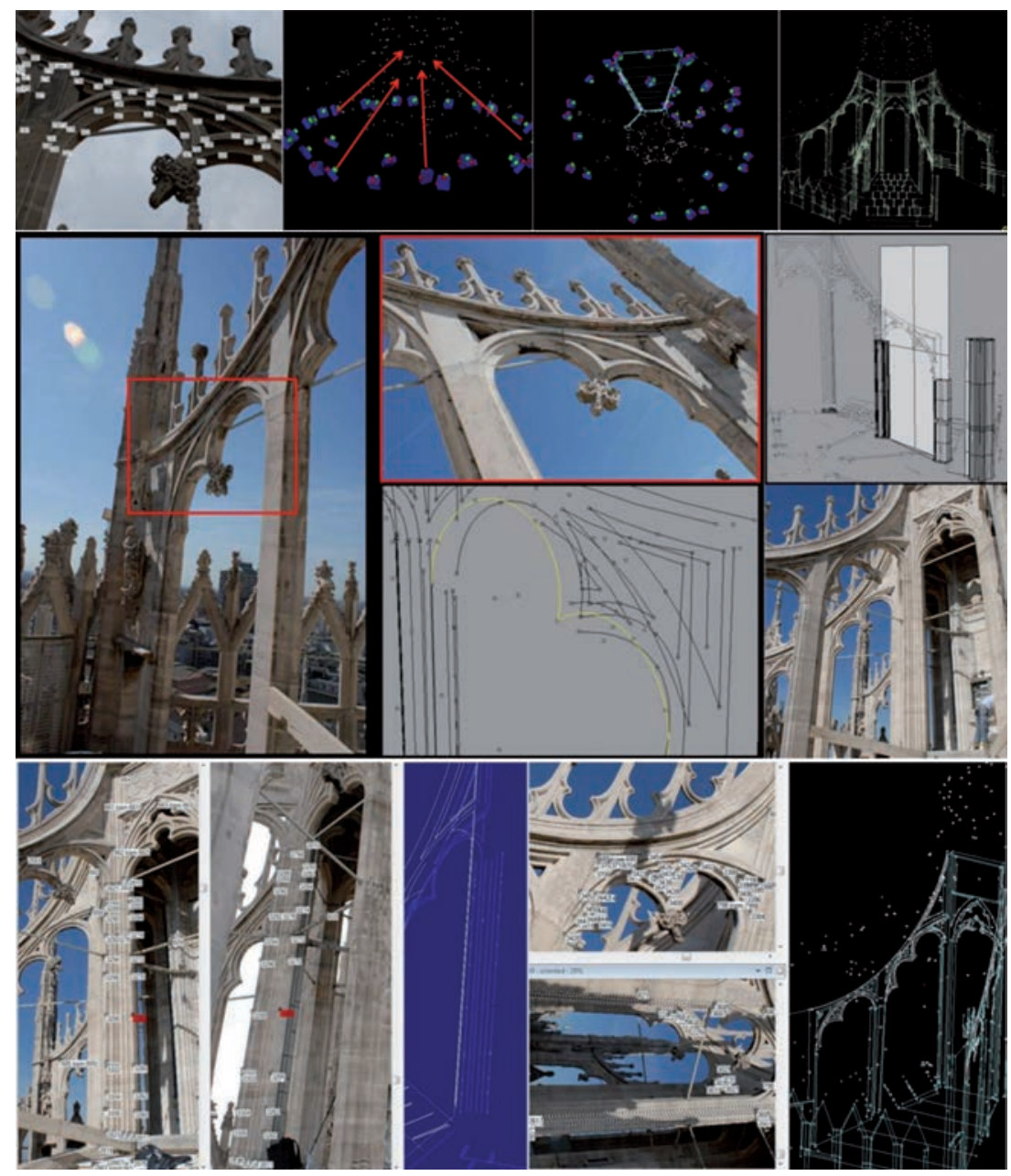

Fig. 4 - Attraverso la restituzione fotogrammetrica è stato possibile ricostruire forma e posizione delle parti lineari della struttura, le immagini illustrano alcuni degli step di costruzione del modello dei pilastri del tiburio. Qui appare l'intervento fotogrammetrico che si rivela fondamentale per l'individuazione di ciascun pezzo della struttura. Qui vengono applicate Tecniche di Image modelling per gli oggetti

"Line based" (scheletro strutturale della guglia) e Tecniche di Image matching per oggetti di tipo"Surface based" (Statue, decorazioni, Guglie secondarie...) acquisendo Nuvole di punti, con passaggio alle Mesh. Per oggetti di tipo "Line based (scheletro della Guglia) acquisendo Nuvole di punti, Estrazione di profili. Il tutto per arrivare alla Modellazione. 


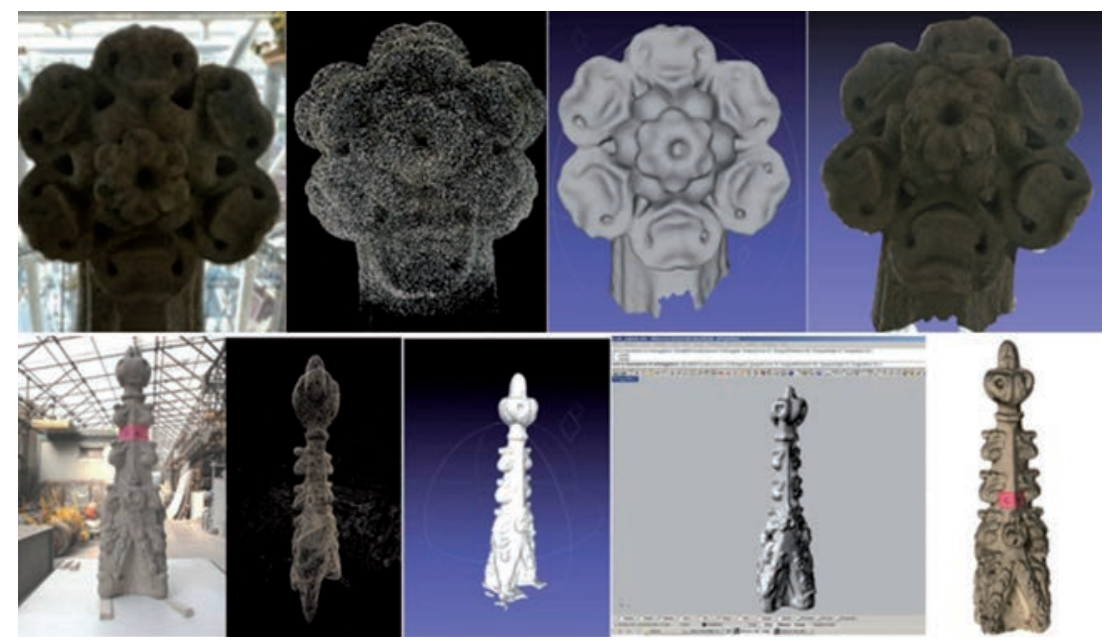

Fig. 5 - Tutti i dettagli relativi agli ornati e statuaria sono stati modellati con la tecnica del matching di immagine. Vale quanto detto per la Fig. 4. Tutti i modelli concorrono alla costruzione del modello generale della Guglia. In rosso si evidenzia il singolo elemento di cui si viene a conoscere il tutto: forma, dimensione, peso. Andando in ordine: dai fotogrammi si estrae la nuvola di punti, si ricava una superficie interpolante, si operano sul modello le correzioni e per ultimo si ha la "vestizione" con le immagini.

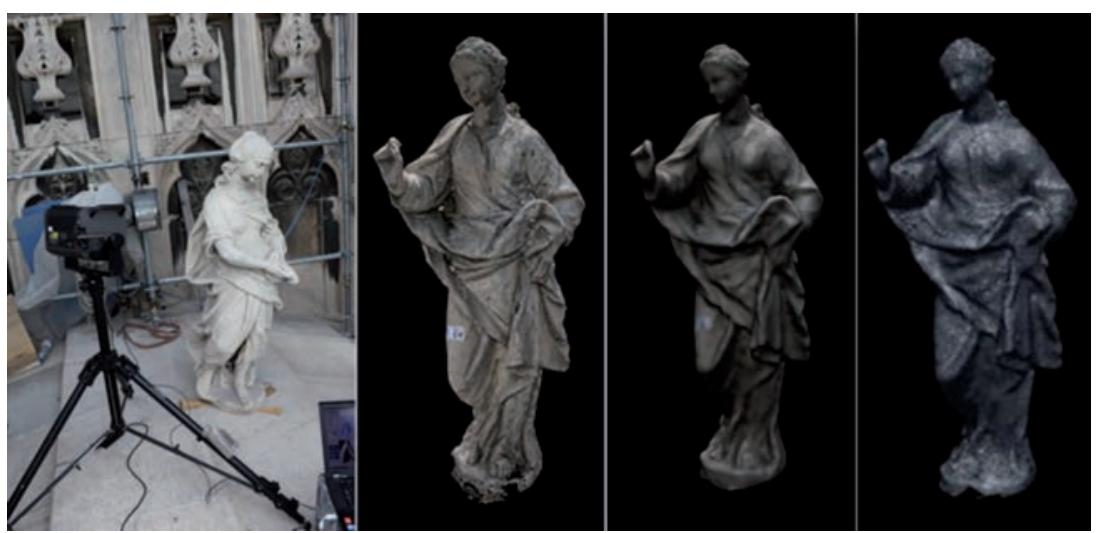

Fig. 6 - Sono state fatte alcune prove di rilievo e modellazione con strumentazione laser scanner (laser triangolatore). Si è utilizzato il Konica Minolta Range7. Questo è uno scanner 3D a triangolazione laser. Per la massima accuratezza, anche su corpo nero, occorrono 96 sec. circa con incertezza sul punto intorno a 0,1 $\mathrm{mm}$.

Ogni scansione acquisisce un massimo di 1.310 .000 punti con un foot print sull'oggetto di circa $20 \times 30 \mathrm{~cm}^{2}$ ad una distanza compresa fra 45 e $80 \mathrm{~cm}$. 


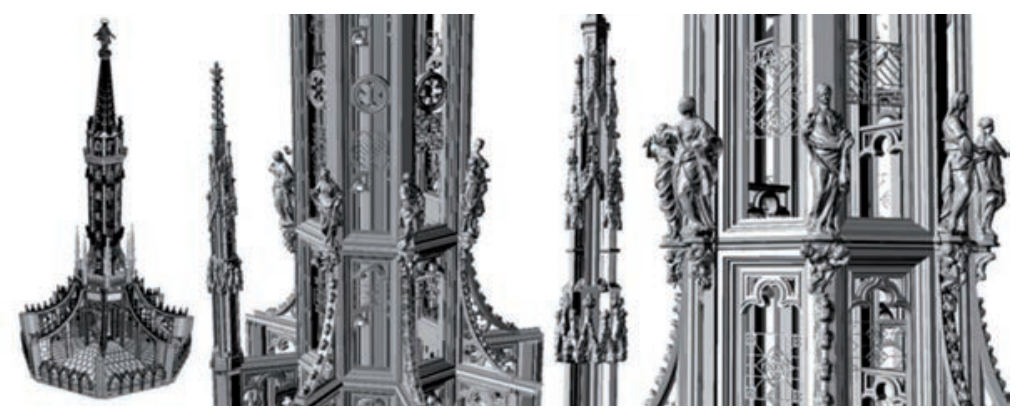

Fig. 7 - Il modello 3D completo della Guglia Maggiore è la 'somma' dei singoli conci, ornati, statue che la compongono. E' possibile esplorare il modello in ogni sua parte....
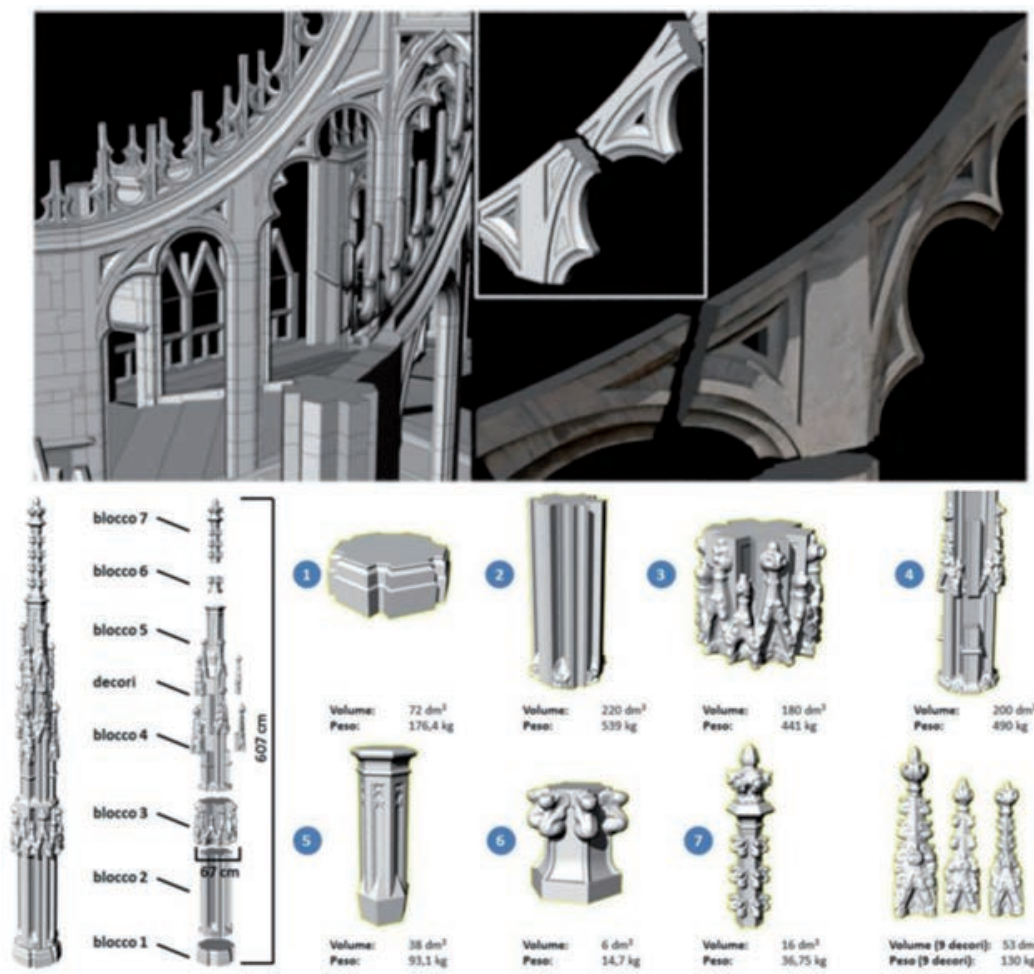

Fig. 8 - Un dettaglio di una delle gugliette che adornano il primo terrazzo.

Sono state modellate tutte le parti (lineari e free form) poi combinate insieme per costruire il modello completo dell'oggetto. Il modello cosi realizzato permette di conoscere le dimensioni delle parti e il peso. Dati particolarmente interessanti per l'ordinaria gestione del cantiere di manutenzione. 

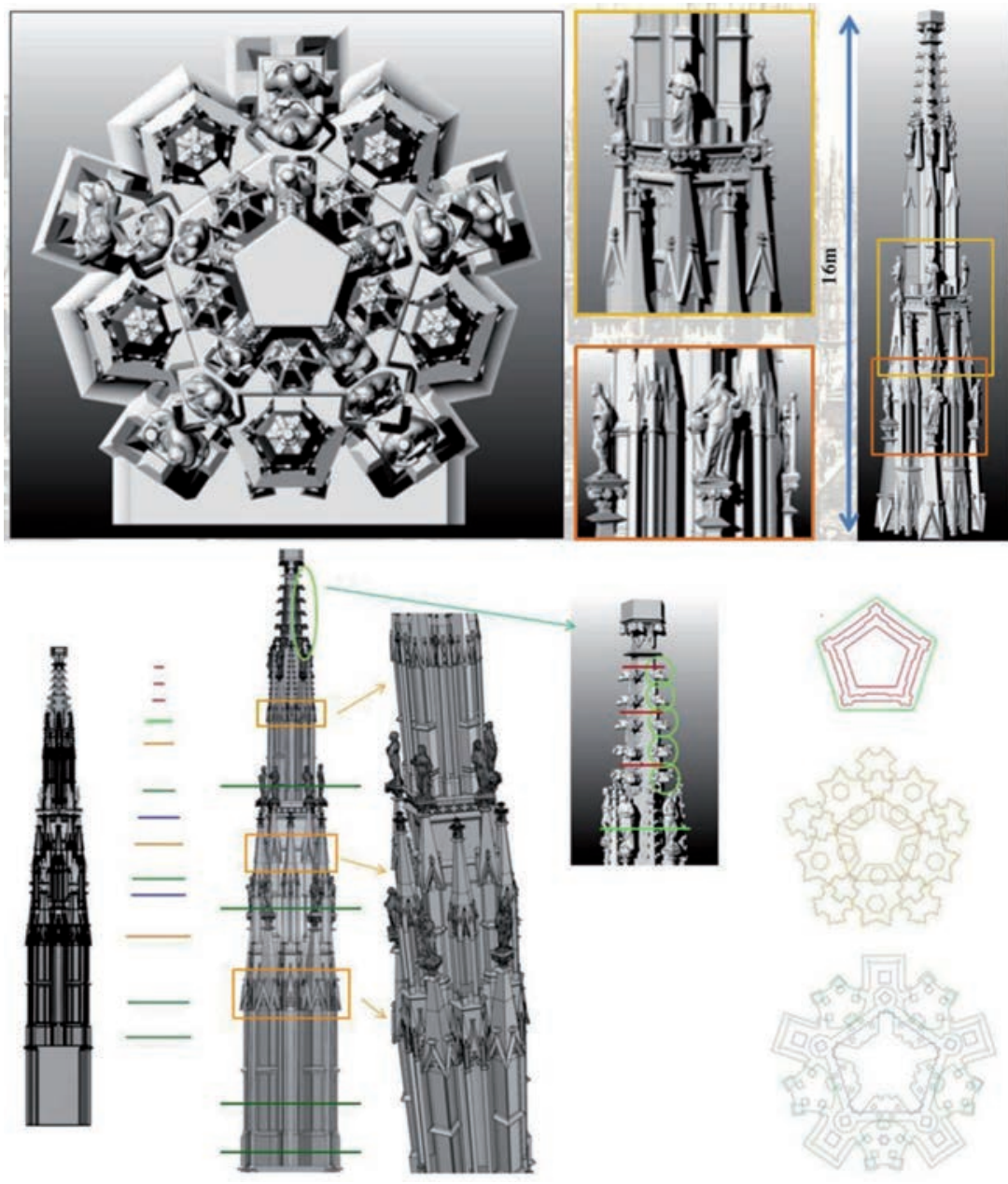

Fig. 9 - La guglia 18. E' l'unica guglia pentagonale del Duomo. E' un tipico esempio di architettura frattale con caratteristiche di omotetia e auto somiglianza, 500 anni prima che Mandelbrot scoprisse e rendesse famosa la geometria frattale. 

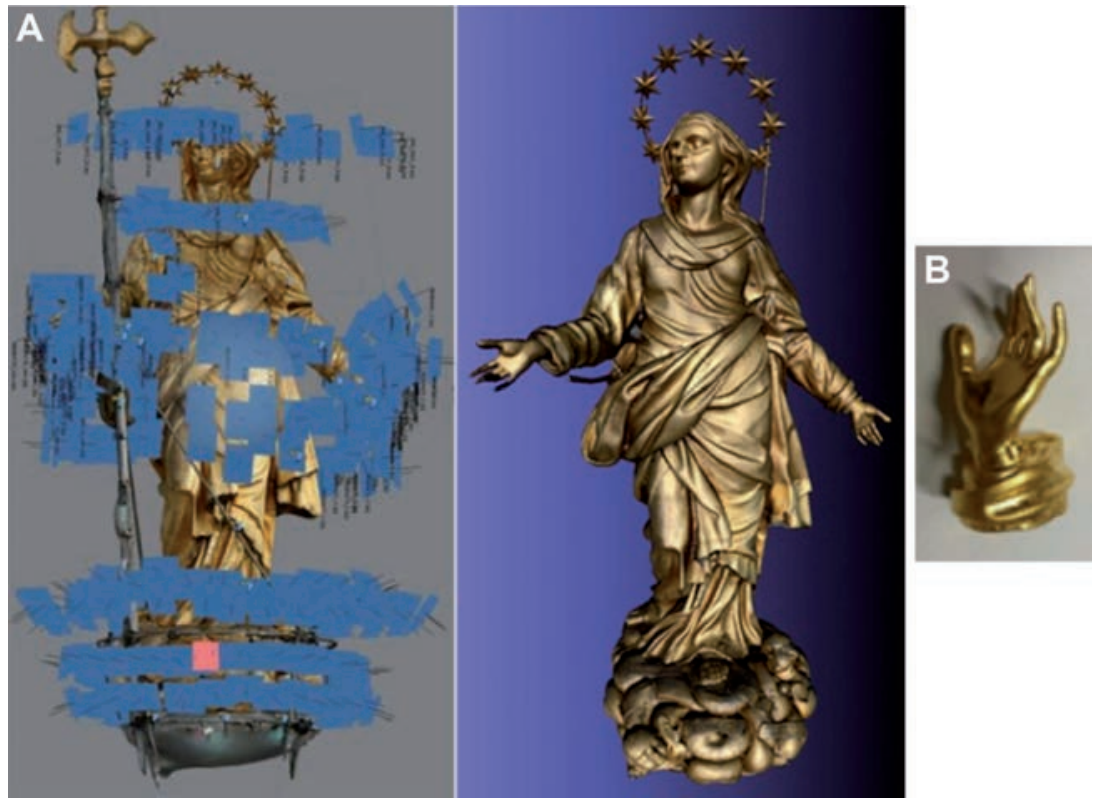

Fig. 10 - A) Il modello della Madonnina. È stata rilevata dopo la ridoratura con una camerera montata su un braccio apposito per raggiungerla in tutta la sua altezza $(4,58 \mathrm{~m})$ E' stata utilizzata una camera CANON 5D MARKIII, Obiettivo $35 \mathrm{~mm}$,

Sensore full frame $24 \times 36 \mathrm{~mm}$. Sono state fatte 458 foto (5760×3840),

11 blocchi fotogrammetrici. Le immagini sono state suddivise in 5 sottogruppi (chunk), dense matching estratto ad alta risoluzione (lim: 20 milioni). Ogni chunck è stato registrato sull'altro usando procedura automatica per la parte bassa del corpo.

Tramite l'utilizzo di target per la parte alta del corpo. Allineamento fine e post-elaborazione della mesch in Rapidform. Numero dei triangoli 155000 circa, risoluzione 0,5 mm. Prototipazione modello in scala 1:5 $(916 \mathrm{~mm}$ ) in resina caricata con alluminio, finitura superficiale di macchina.

B) Finitura finale in foglia d'oro della mano destra. 

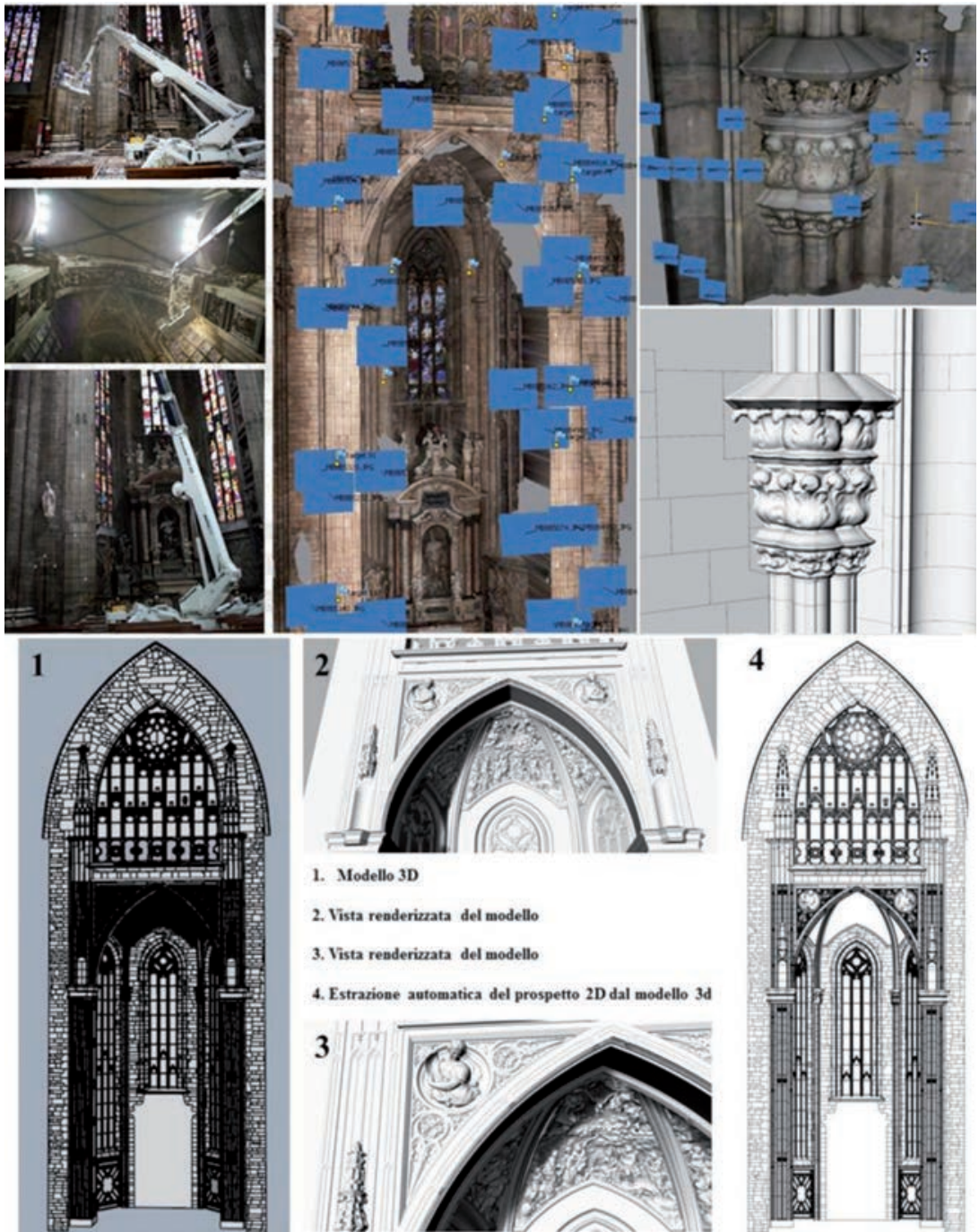

1. Modello 3D

2. Vista renderimata del modello

3. Vista readerizata del modello

4. Estrarione automatica del prospetto $2 \mathrm{D}$ dal modello $3 \mathrm{~d}$
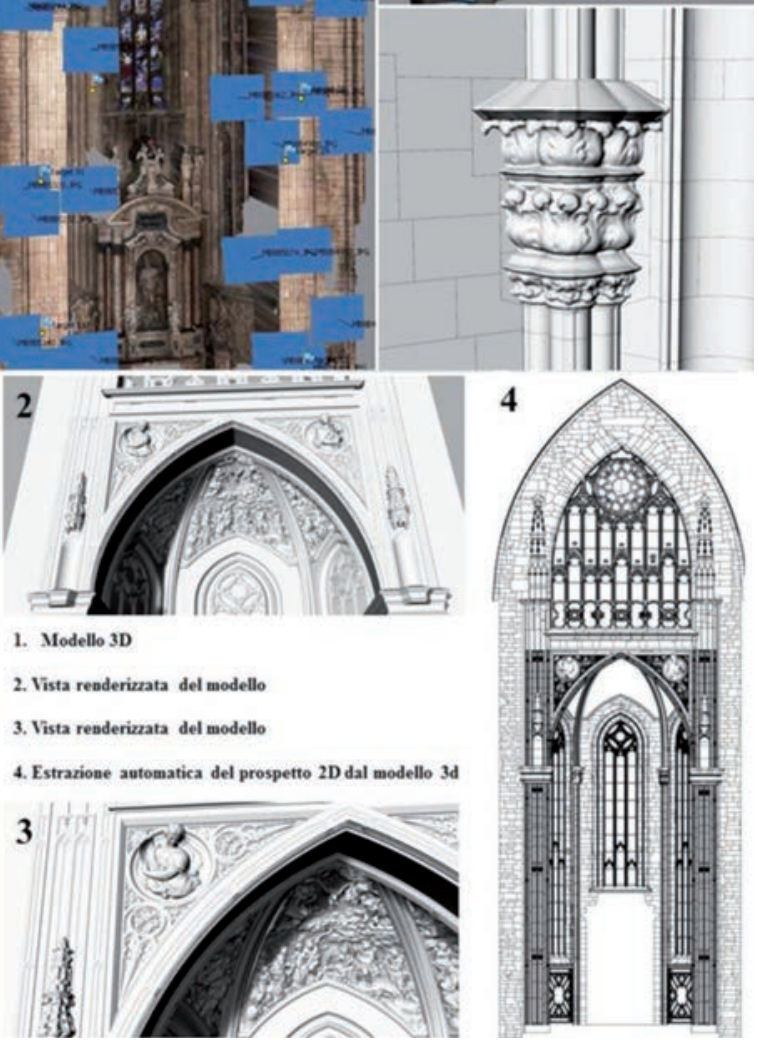

Fig. 11 - Anche gli altari posti ai lati del transetto sono stati rilevati con tecnica fotogrammetrica. Dalle immagini acquisite alle diverse quote è stato possibile ricavare il modello 3D, poi scomposto nei conci costruttivi. Dal modello 3D è sempre possibile estrarre qualunque vista $2 D$ scegliendo di volta in volta quella necessarie in funzione delle esigenze dell'ufficio tecnico e del cantiere. 

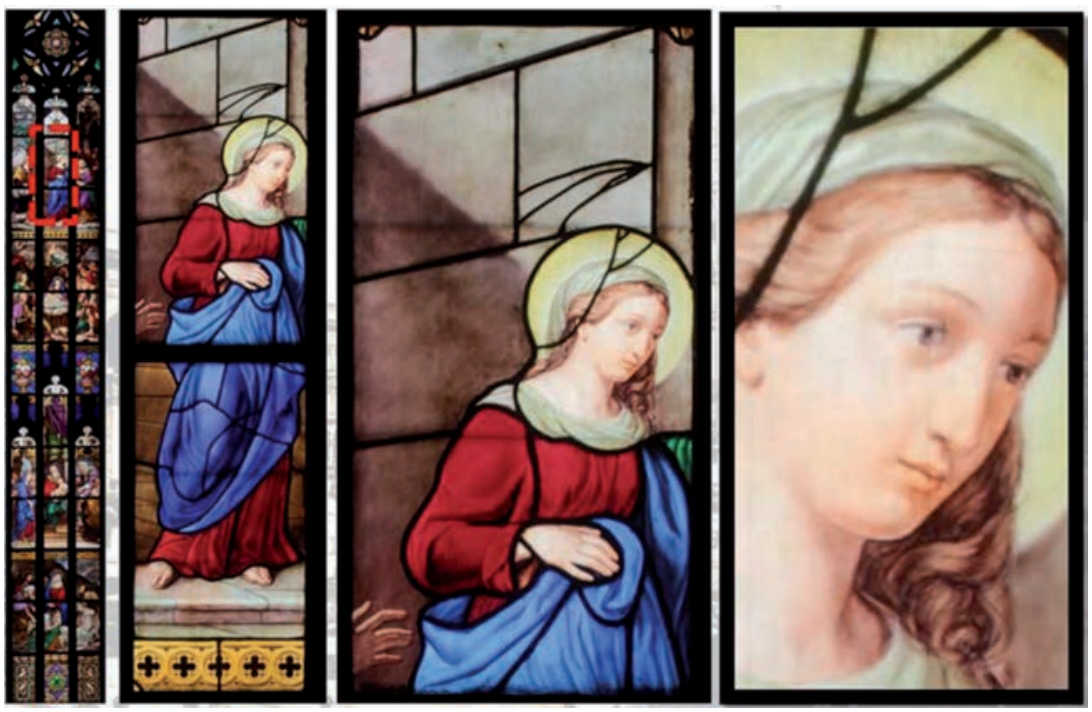

Fig. 12 - Sono state realizzate anche le ortofoto delle magnifiche vetrate che adornano gli altari, l'immagine illustra un particolare. 